\title{
Patterns of locoregional failure following post-operative intensity-modulated radiotherapy to oral cavity cancer: quantitative spatial and dosimetric analysis using a deformable image registration workflow
}

Abdallah S. R. Mohamed ${ }^{1,3^{*}}$, Andrew J. Wong ${ }^{1}$, Clifton D. Fuller ${ }^{1}$, Mona Kamal ${ }^{1,4}$, Gary B. Gunn ${ }^{1}$, Jack Phan ${ }^{1}$, William H. Morrison ${ }^{1}$, Beth M. Beadle ${ }^{1}$, Heath Skinner ${ }^{1}$, Stephen Y. Lai ${ }^{2}$, Sean R. Quinlan-Davidson ${ }^{1,5}$, Abdelaziz M. Belal ${ }^{3}$, Ahmed G. El-Gowily ${ }^{3}$, Steven J. Frank1', David I. Rosenthal ${ }^{1}$ and Adam S. Garden ${ }^{\text {* }}$

\begin{abstract}
Background: We sought to identify spatial/dosimetric patterns of failure for oral cavity cancer patients receiving post-operative IMRT (PO-IMRT).

Methods: Two hundred eighty-nine OCC patients receiving PO-IMRT were retrospectively reviewed from 2000 to 2012. Diagnostic $C T$ documenting recurrence $(r C T)$ was co-registered with planning $C T(p C T)$ using a validated deformable image registration software. Manually segmented recurrent gross disease ( $\mathrm{G}$ TV) was deformed to co-registered pCTs. Mapped rGTVs were compared dosimetrically to planned dose and spatially to planning target volumes using centroidbased approaches. Failures types were classified using combined spatial/dosimetric criteria: A (central high-dose), B (peripheral high-dose), C (central intermediate/low-dose), D (peripheral intermediate/low-dose), and E (extraneous-dose).

Results: Fifty-four patients with recurrence were analyzed; 26 local recurrence, 19 regional recurrence, and 9 both local and regional recurrence. Median time to recurrence was 4 months (range 0-71). Median rGTVs volume was $3.7 \mathrm{~cm}^{3}$ (IQR 1.4-10.6). For spatial and dosimetric analysis of the patterns of failure, 30 patients (55.5\%) were classified as type A (central high-dose). Non-central high dose failures were distributed as follows: 2 (3.7\%) type B, 10 (18.5\%) type C, 1 (1.8\%) type D, and 9 (16.7\%) type E. Non-IMRT failure in the matching low-neck field was seen in two patients. No failures were noted at the IMRT-supraclavicular field match-line.

Conclusions: Approximately half of patients with local/regional failure had non-central high dose recurrence. Peripheral high dose misses were uncommon reflecting adequate delineation and dose delivery. Future strategies are needed to reduce types $C$ and $E$ failures.
\end{abstract}

Keywords: Patterns of failure, Post-operative intensity modulated radiation therapy, Oral cavity cancer, Deformable image registration, Quantitative spatial and dosimetric analysis

\footnotetext{
* Correspondence: asmohamed@mdanderson.org; agarden@mdanderson.org 1 Departments of Radiation Oncology, The University of Texas MD Anderson Cancer Center, Unit 97, 1515 Holcombe Boulevard, Houston, TX 77030, USA Full list of author information is available at the end of the article
} 


\section{Introduction}

Surgery is often the treatment of choice for oral cavity squamous cell carcinoma (OCSCC). Post-operative radiotherapy is indicated for OCSCC of advanced stages or with adverse prognostic factors [1-3]. Intensitymodulated radiotherapy (IMRT) enables conformal therapy and reduction of complications to surrounding normal tissue, and for many centers has become the standard radiation approach for head and neck cancer [4].

Generally, OCSCC patients demonstrate relatively worse loco-regional control compared to other head and neck subsites (e.g. oropharynx and larynx) [5-7]. Studies that have specifically examined cohorts of OCSCC patients receiving postoperative IMRT (PO-IMRT) have consistently reported only fair locoregional control rates, as low as $53 \%$ at 3 years in some series [8-13].

Moreover, most report failures as "infield", "marginal", or "outfield" based on percentage overlap between failure volume and respective target volumes. However, these studies applied non-uniform spatial methods for failure analysis, mainly utilizing non-validated rigid or manual image registration tools and without including the dosimetric component in the analysis [8-13]. We have recently shown the potential impact of patterns of failure analysis methodology using a validated image registration software paired with combined spatial and dosimetric analysis of failure, in improving the accuracy of reporting the patterns of failure in the era of IMRT [14-16]. As a continuation of these efforts we sought to apply this unique analytic methodology to our institutional large scale oral cavity cancer dataset of patients receiving PO-IMRT with documented treatment failure to achieve the following specific aims: 1) characterize distinct spatial and dosimetric patterns of failure after PO-IMRT, 2) identify clinical risk features associated with each failure type, 3) identify patterns of failure based target volume contouring recommendations, and 4) generate hypotheses for future clinical trials.

\section{Material and methods \\ Patient selection}

Two hundred eighty-nine patients with pathological diagnosis of OCC who received PO-IMRT at the University of Texas MD Anderson Cancer Center from 2000 to 2012 were retrospectively reviewed under an approved institutional review board protocol. Patients with distant metastases or concurrent malignancies at the time of diagnosis, or treatment with chemotherapy prior to staging at MDACC were excluded. Patients with prove of recurrence after PO-IMRT with available imaging documenting recurrence were included in the current analysis.

\section{Treatment planning and delivery}

Treatment planning and delivery is described in details in previously published work, which examines the outcomes for this same patient cohort [17].

\section{Clinical data collection}

Diagnostic contrast-enhanced CT and/or PET/CT documenting the initial evidence of local and/or regional recurrence $(\mathrm{rCT})$ was identified. Recurrences were confirmed via radiologic imaging (i.e. progression in subsequent CT imaging or high SUV on PET imaging) or pathology specimens (i.e. from surgical biopsy). Radiologically evident recurrent gross disease (rGTV) was manually segmented and reviewed by two experienced radiation oncologists (ASRM, CDF). Corresponding original planning CTs $(\mathrm{pCT})$ were also identified and original plans were restored. Patient, disease, and treatment characteristics were gathered during chart review.

\section{Image registration and dosimetric analysis}

rCT was co-registered with $\mathrm{pCT}$ using a previously validated deformable image registration (DIR) methodology (VelocityAI 3.0.1, Velocity Medical Solutions, Atlanta, GA, 2004-2013) [14, 15]. rGTVs on the rCT were subsequently deformed to co-registered pCT (Fig. 1).

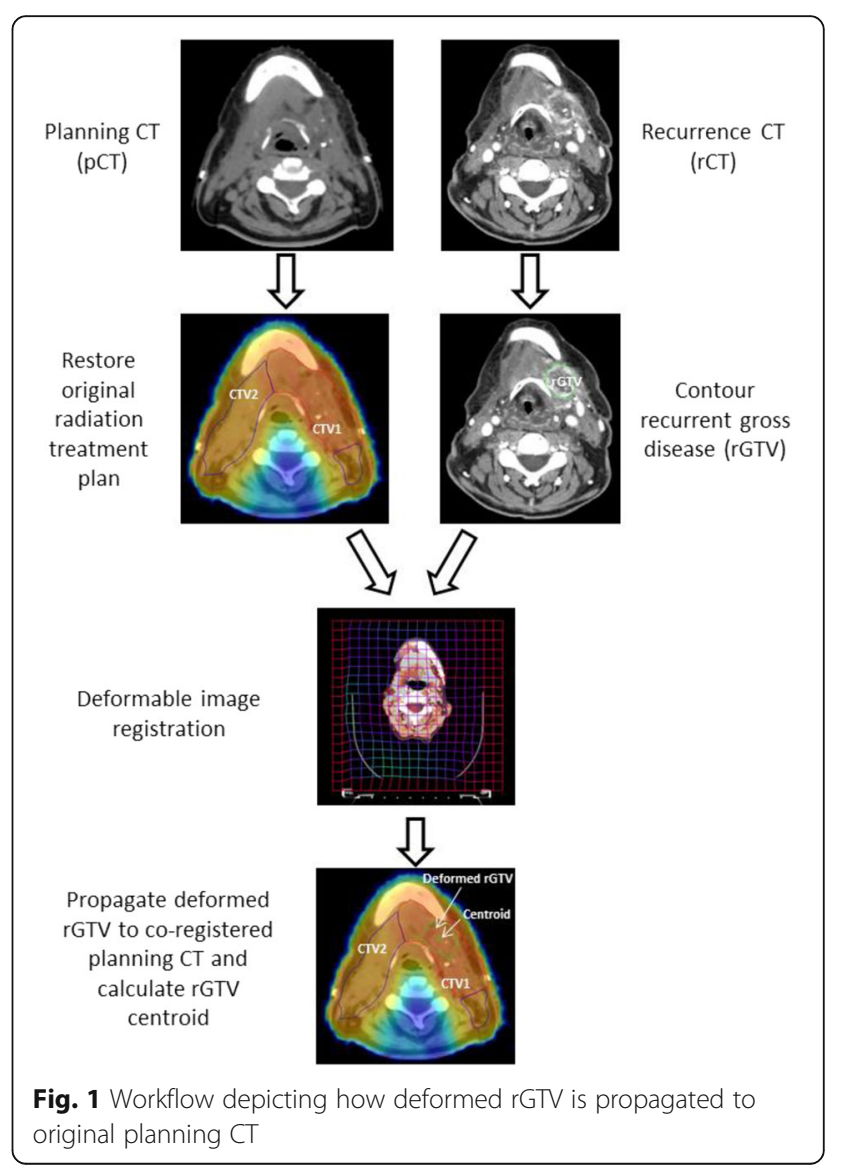


The centroid, assumed as the origin of tumor recurrence, is represented as the calculated center of mass of the deformed rGTV. Dosimetric and volumetric parameters were obtained from the dose-volume histogram.

\section{Patterns of failure classification}

Failures are classified according to both spatial and dosimetric criteria as previously described. [16] Briefly, for spatial mapping of recurrence origin, the centroid of each rGTV was mapped to the corresponding TV in the planning CT. Subsequently, the dosimetric characteristics were assessed by calculating the dose to $95 \%$ of the failure volume (fD95\%) then comparing it relative to the dose prescribed to the corresponding TV of origin as determined by the spatial mapping. Finally, failures were classified into five major types: Type A (central high dose where the mapped failure centroid originates in high dose TV and $\mathrm{fD} 95 \%$ is $\geq 95 \%$ dose prescribed to corresponding high dose TV of origin), Type B (peripheral high dose where the failure centroid originates from high dose TV but its fD95\% is $<95 \%$ dose prescribed to corresponding high dose TV of origin), Type $\mathrm{C}$ (central elective dose where the failure centroid originates in lower dose TV and $\mathrm{fD} 95 \%$ is $\geq 95 \%$ dose prescribed to corresponding lower dose TV of origin), Type D (peripheral elective dose where the failure centroid originates in lower dose TV but the $\mathrm{fD} 95 \%$ is $<95 \%$ dose prescribed to corresponding lower dose TV of origin), and Type E (extraneous dose where rGTV centroid originates outside all TVs). Type F describes junctional failures at the IMRT/supraclavicular match line, and Type $G$ describes low neck failures at the low-neck supraclavicular field. Type $\mathrm{G}$ is analogous to type $\mathrm{C}$ if the $\mathrm{fD} 95 \%$ is $\geq 95 \%$ dose prescribed to the low-neck and analogous to type D if the fD95\% is $<95 \%$. Examples demonstrating failure type definitions are illustrated in Fig. 2.

Patients were then classified according to the predominant mode of failure. Patients with more than single recurrence lesion were classified as the following: 1) for patients with type A recurrence and concurrent nontype A lesions, the overall pattern of failure was defined as type A because we believe type A for such patients is the true recurrence rather than reseeding from the nontype A recurrence, 2) for patients whom exhibited more than one failure type of non-type A simultaneously, pattern of failure of each patient was classified according to the predominant type as determined by the most commonly encountered failure type (i.e. higher number or higher volume).

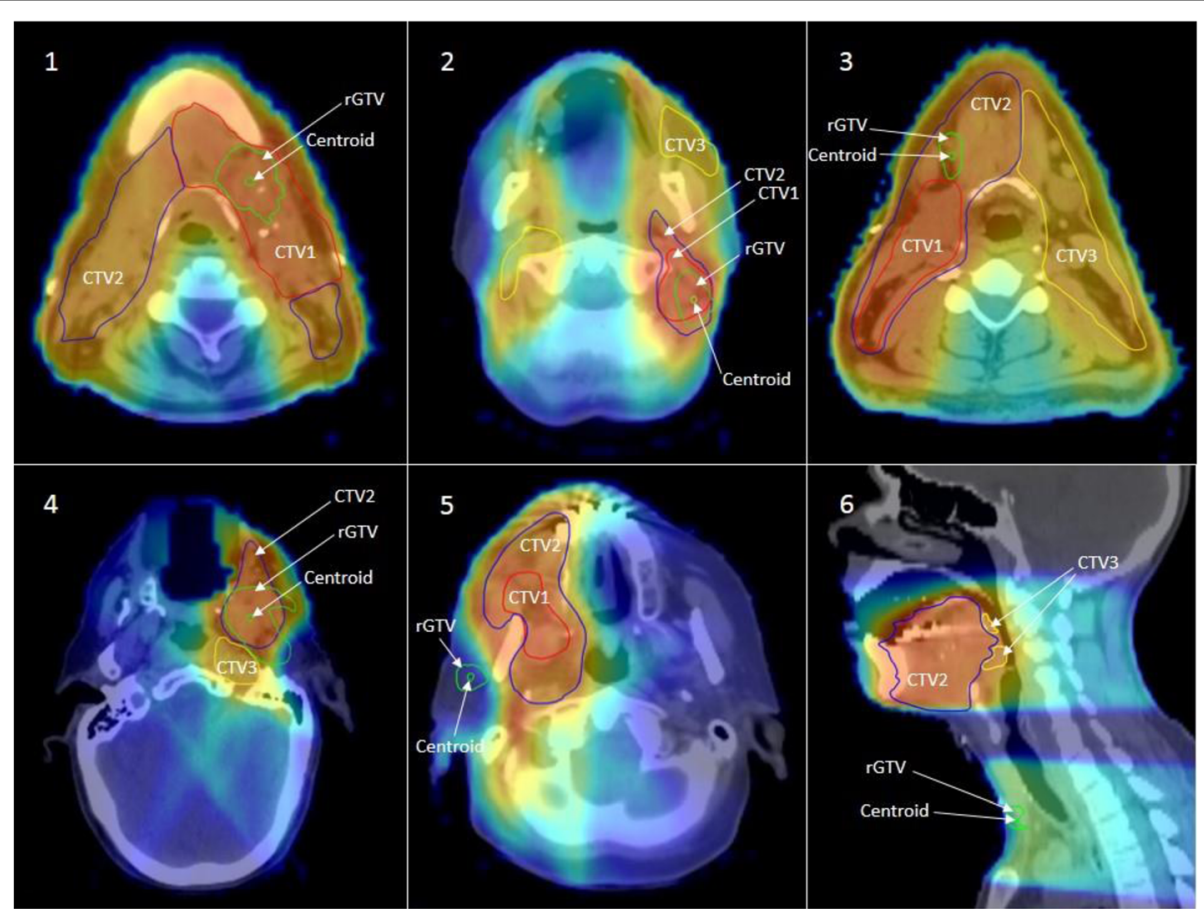

Fig. 2 Examples of Failure Types. 1) Type A (central high dose) failures. Centroid is mapped inside high dose TV and dose to 95\% rGTV volume $\geq 95 \%$ dose prescribed to high dose TV. 2) Type B (peripheral high dose) failure. Centroid is mapped inside high dose TV, but dose to $95 \%$ rGTV volume $<95 \%$ dose prescribed to high dose TV. 3) Type C-int (central intermediate dose) failure. Centroid is mapped inside intermediate dose TV and dose to $95 \%$ rGTV volume $\geq 95 \%$ dose prescribed to intermediate dose TV. 4) Type D-int (peripheral intermediate dose) failure. Centroid is mapped inside intermediate dose TV but dose to 95\% rGTV volume < 95\% dose prescribed to intermediate dose TV. 5) Type E (Extraneous dose failure) where rGTV centroid originates outside all TVs. 6) Type G (low neck failure) where rGTV centroid is located at the low-neck supraclavicular field 


\section{Results}

\section{Patient and treatment characteristics}

Sixty-three patients (22\%) developed locoregional recurrences. Median time to recurrence was 4 months (range 0-71). For spatial and dosimetric analysis of the patterns of failure, 9 patients were excluded: 4 with post-surgical recurrence prior to initiation of IMRT, 3 with no retrievable IMRT plan, and 2 with no available imaging documenting recurrence. This left 54 patients for the current analysis.

Patient, disease, and treatment characteristics for the analyzed 54 patients are summarized in Table 1 . The most common primary site was the oral tongue (39\%). The most common pathologic $\mathrm{T}$ and $\mathrm{N}$ staging were $\mathrm{T} 2$ (37\%) and N2 (36\%). Forty-seven (87\%) patients had Stage III-IV disease.

Surgical margins were positive and close (defined as $\leq 5 \mathrm{~mm})$ in $4(7 \%)$ and $9(17 \%)$ patients, respectively. Perineural invasion or lymphovascular invasion was present in $22(41 \%)$ and $14(26 \%)$ patients, respectively. Depth of invasion was $\geq 1.5 \mathrm{~cm}$ in 18 patients (33\%). Forty-seven patients (87\%) had neck dissections, and of those 17 patients had nodal extracapsular extension.

Mean RT dose was $60 \pm 7$ Gy and mean number of fractions was $30 \pm 3$. One patient did not complete the full course of IMRT, discontinuing therapy after 6 fractions. Thirteen (24\%) and $41(76 \%)$ patients received unilateral and bilateral neck irradiation, respectively. Mean overall treatment package time, defined as time interval from date of surgery to last day of irradiation, was $12.3 \pm 1.7$ weeks.

\section{Recurrence characteristics}

For patients included in the current analysis; 26 (48\%) had local recurrence, 19 (35\%) had regional recurrence, and $9(17 \%)$ had both local and regional recurrence. A total of 82 rGTVs were delineated. Median rGTVs volume was $3.7 \mathrm{~cm}^{3}$ (IQR 1.4-10.6). Figure 3 shows the distribution of the predominant type of failure for the entire dataset using the proposed classification schema. Thirty patients $(55.5 \%)$ were classified as type A. Nontype A failures were distributed as follows: 2 (3.7\%) type B, 10 (18.5\%) type C, 1 (1.8\%) type D, 9 (16.7\%) type E, and $2(3.7 \%)$ type G. Because type A "central high dose" failures, are considered to resistance to maximal therapy, and thus could not conceivably be prevented by technical/operator dependent processes, however, nontype A failures represent a major goal for IMRT quality assurance and improvement. Table 2 illustrates the details of the characteristics for all non-type A failures.

\section{Local failure typology}

Of the 26 patients with local disease failure without synchronous regional recurrence, 16 (62\%) were type A central high dose failures. Ten patients (38\%) had nontype A local failure; two (8\%) were type B, three (11\%) were type C (intermediate dose), one (4\%) was type D (intermediate dose), and four (15\%) were extraneous type E failure. Three of patients with type A failure had multifocal recurrence; two patients with two foci of recurrences (both within the central high dose region), and one had four foci of recurrences (three in the intermediate dose and one in the high dose).

For the two patients with type B failure, one had a primary tumor in the left mandibular gingiva and developed recurrence involving the left maxillary sinus, with erosion of its lateral wall and extension along the buccal mucosa to the retromolar trigone. The second patient had a primary tumor in the floor of mouth with rapid disease progression subsequent to discontinuing radiation treatment after six fractions.

Regarding patients with type $C$ failure, one patient had his primary tumor in the retromolar area and recurrence in the ipsilateral masticator space. The second patient had the primary tumor in the floor of mouth with recurrence involving the base of tongue, while the third patient had a primary tumor in the oral tongue with recurrence involving the floor of mouth.

The single type D failure had the primary tumor in the left mandibular gingiva and recurred along the left masticator space allowing the rGTV to partially grow outside the CTV2 boundaries, however the centroid was still located inside CTV2.

For patients with type $\mathrm{E}$ failure, one had the primary tumor in the right mandibular gingiva and the recurrence in the contralateral mandibular gingiva approximately 2 years following treatment. The second patient had the primary tumor in the left buccal mucosa; the recurrence manifested as retrograde perineural spread that extended to the left pterygopalatine fossa, foramen rotundum, foramen ovale, cavernous sinus, and through the superior orbital fissure into the left orbit (Fig. 4). The third patient had T1 primary tumor in the central floor of mouth and the recurrence in the left alveolar mandibular ridge approximately 3 years following treatment. Lastly, the fourth patient had the primary tumor in the left mandibular gingiva and the recurrence in the ipsilateral masticator space at the first follow up following treatment.

\section{Regional failure typology}

Of the 19 patients with regional disease failure without synchronous local recurrence, only 7 patients (37\%) were type A central high dose failures. Twelve patients (63\%) had non-type A local failure; 5 (26\%) were type $C$ (intermediate or low dose), five (26\%) were extraneous type E, and $2(11 \%)$ were type $G$ low neck failure. Two of patients with type A failure had multifocal recurrence. One 
Table 1 Patient, disease, and treatment characteristics

\begin{tabular}{|c|c|}
\hline Characteristic & N (\%) \\
\hline \multicolumn{2}{|l|}{ Age } \\
\hline Median (range) & 59.5 years $(22-87)$ \\
\hline \multicolumn{2}{|l|}{ Gender } \\
\hline Female & $20(37)$ \\
\hline Male & $34(63)$ \\
\hline \multicolumn{2}{|l|}{ Tumor Site } \\
\hline Oral Tongue & $21(39)$ \\
\hline Buccal Mucosa & $10(18.5)$ \\
\hline Floor of Mouth & $2(4)$ \\
\hline Hard Palate & $3(5)$ \\
\hline Gingiva & $10(18.5)$ \\
\hline Retromolar Trigone & $8(15)$ \\
\hline \multicolumn{2}{|c|}{ Histologic Differentiation } \\
\hline Poor & $13(24)$ \\
\hline Moderate & $36(67)$ \\
\hline Well & $5(9)$ \\
\hline \multicolumn{2}{|l|}{ Clinical T stage } \\
\hline $\mathrm{T} 1$ & $8(15)$ \\
\hline $\mathrm{T} 2$ & $19(35)$ \\
\hline T3 & $9(17)$ \\
\hline $\mathrm{T} 4$ & $18(33)$ \\
\hline \multicolumn{2}{|l|}{ Clinical N stage } \\
\hline $\mathrm{Nx}$ & $2(4)$ \\
\hline NO & $23(43)$ \\
\hline N1 & $11(20)$ \\
\hline $\mathrm{N} 2 \mathrm{a}$ & $0(0)$ \\
\hline $\mathrm{N} 2 \mathrm{~b}$ & $14(26)$ \\
\hline $\mathrm{N} 2 \mathrm{C}$ & $4(7)$ \\
\hline \multicolumn{2}{|l|}{ Pathological T stage } \\
\hline урт0 & $2(4)$ \\
\hline T1 & $8(15)$ \\
\hline $\mathrm{T} 2$ & $20(37)$ \\
\hline T3 & $7(13)$ \\
\hline $\mathrm{T} 4$ & $17(31)$ \\
\hline \multicolumn{2}{|l|}{ Pathological N stage } \\
\hline No dissection & $7(13)$ \\
\hline NO & $12(22)$ \\
\hline N1 & $12(22)$ \\
\hline $\mathrm{N} 2 \mathrm{a}$ & $0(0)$ \\
\hline $\mathrm{N} 2 \mathrm{~b}$ & $19(36)$ \\
\hline $\mathrm{N} 2 \mathrm{C}$ & $4(7)$ \\
\hline \multicolumn{2}{|l|}{ Overall stage } \\
\hline Stage I & $1(2)$ \\
\hline Stage II & $6(11)$ \\
\hline
\end{tabular}

Table 1 Patient, disease, and treatment characteristics (Continued)

\begin{tabular}{|c|c|}
\hline Stage III & $7(13)$ \\
\hline Stage IV & $40(74)$ \\
\hline \multicolumn{2}{|l|}{ Primary Surgery Margin Status } \\
\hline Negative (>5 mm) & $41(76)$ \\
\hline Close $(\leq 5 \mathrm{~mm})$ & $9(17)$ \\
\hline Positive & $4(7)$ \\
\hline \multicolumn{2}{|l|}{ Depth of invasion } \\
\hline$\leq 1.5 \mathrm{~cm}$ & $33(61)$ \\
\hline$>1.5 \mathrm{~cm}$ & $18(33)$ \\
\hline Unspecified & $3(6)$ \\
\hline \multicolumn{2}{|l|}{ Perineural invasion } \\
\hline Yes & $22(41)$ \\
\hline No & $32(59)$ \\
\hline \multicolumn{2}{|l|}{ Lymphovascular invasion } \\
\hline Yes & $14(26)$ \\
\hline No & $30(56)$ \\
\hline Unspecified & $10(18)$ \\
\hline \multicolumn{2}{|l|}{ Extracapsular extension } \\
\hline Yes & $17(31)$ \\
\hline No & $37(69)$ \\
\hline \multicolumn{2}{|l|}{ IMRT dose and fractionation } \\
\hline Median Dose (Range), in Gy & $60(56-70)$ \\
\hline Median Fractionation (Range) & $30(28-33)$ \\
\hline \multicolumn{2}{|l|}{ Laterality of Neck radiation } \\
\hline Unilateral & $13(24)$ \\
\hline Bilateral & $41(76)$ \\
\hline \multicolumn{2}{|l|}{ Chemotherapy } \\
\hline Induction & $5(9)$ \\
\hline Concurrent & $13(24)$ \\
\hline Induction and concurrent & $2(4)$ \\
\hline No chemotherapy & $32(59)$ \\
\hline
\end{tabular}

patient with a left oral tongue primary developed synchronous ipsilateral type A recurrence at level III and contralateral type $\mathrm{C}$ (low dose) recurrence at level IIa. The second patient had the primary disease in the right retromolar trigone with multi-nodal recurrence at ipsilateral neck level IIb and an ipsilateral retropharyngeal lymph node (type D).

For the five patients with type $\mathrm{C}$ failure, one patient had a right hard palate primary with multifocal type $C$ (low dose) failure with two foci of recurrence, both at contralateral level IIa. The second patient had the primary tumor in the left maxillary ridge and recurred in the low dose region at the ipsilateral level Ib. The third and fourth patients had primary tumors of the oral 


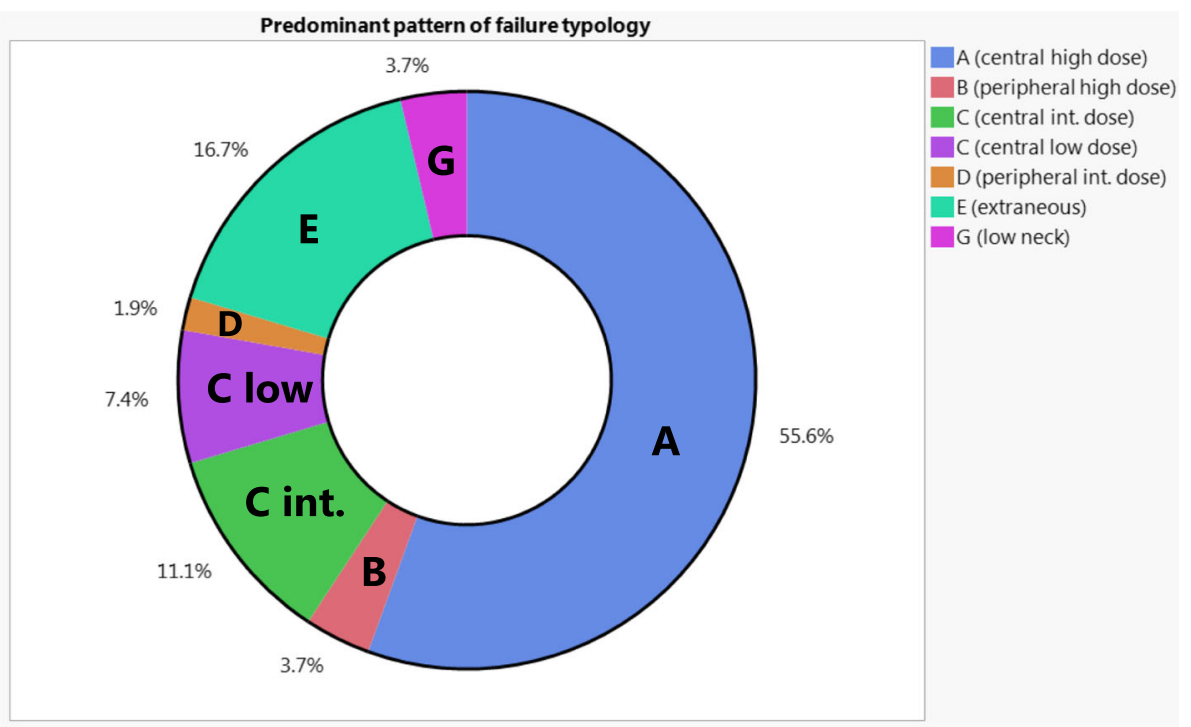

Fig. 3 Ring chart that depicts the distribution of the predominant typology of failure for the entire dataset $(n=54)$

tongue with recurrences in levels Ia (intermediate dose) and contralateral Ib (low dose), respectively. The fifth patient had a primary tumor in the right retromolar trigone and recurrence in the ipsilateral level Ib (intermediate dose).

Four of the five patients with type E extraneous failure had their primary tumor in the buccal mucosa. Two patients had ipsilateral parotid nodal recurrence in the area of spared parotid gland (Fig. 4) while the other two patients had recurrences in the un-irradiated contralateral side. The fifth patient had the primary tumor in the left maxillary alveolar ridge with recurrence in the unirradiated contralateral level II.

Regarding the two patients with type G low neck failure, one had the primary tumor in the left mandibular with negative dissection of ipsilateral neck levels II, III, and IV, however, the patient then recurred in the ipsilateral level VIb (i.e. pre-tracheal recurrence). This failure is analogous to type D as $\mathrm{fD} 95 \%$ for this patient was 10 Gy (i.e. fD $95 \%$ had less than $95 \%$ of the dose prescribed to left supraclavicular region which was $58 \mathrm{~Gy}$ ). The second patient had the primary tumor in the left oral tongue with positive neck dissection of ipsilateral levels I, II, and V then recurred in the contralateral levels III and IV. This failure is analogous to type $\mathrm{C}$ as fD95\% for this patient was 48.75 Gy (i.e. fD $95 \%$ had higher than $95 \%$ of the dose prescribed to contralateral supraclavicular field that encompassed both contralateral levels III and IV with a prescription dose of 50 Gy).

\section{Locoregional failure typology}

Nine patients had synchronous local and regional failure. The predominant typology for each patient was determined according to the local failure component. Seven (78\%) had type A failure and two (22\%) had type C. For patients with type A failure, 3 had synchronous non-type-A failure at the neck ( 2 had synchronous type $\mathrm{C}$ and one had synchronous type $\mathrm{G})$. Both patients with type $\mathrm{C}$ failure had their primary tumor in the oral tongue. The first had recurrence in the contralateral side of the tongue and neck level II (both at low dose CTV) while the second had recurrence in the floor of mouth (intermediate dose CTV) and contralateral neck level IIa (low dose CTV).

\section{Discussion}

Our results demonstrate that nearly half of the patients with local and/or regional failure included in the current study had non-central high dose recurrence. We applied our novel patterns of failure analysis and classification methods to further analyze those recurrences of noncentral high dose nature. Recurrences in operated tissues are less prone to present concentrically as do recurrences that occur following definitive radiation. The likelihood of a recurrence manifesting in the epicenter of origin disease that is removed surgically is unlikely, particularly when large volumes of tissue are removed and replaced by free flaps, creating significant variations in the irradiated anatomy compared to the presurgical anatomy. Furthermore, the new tissue planes can create differing paths for tumor to spread through. Thus in the postoperative setting we essentially create a crude probabilistic model of where microscopic disease may be hiding.

While concentric central recurrences occurred less commonly than we noted in the definitive setting, over $75 \%$ of recurrences did occur within targeted tissues, the 


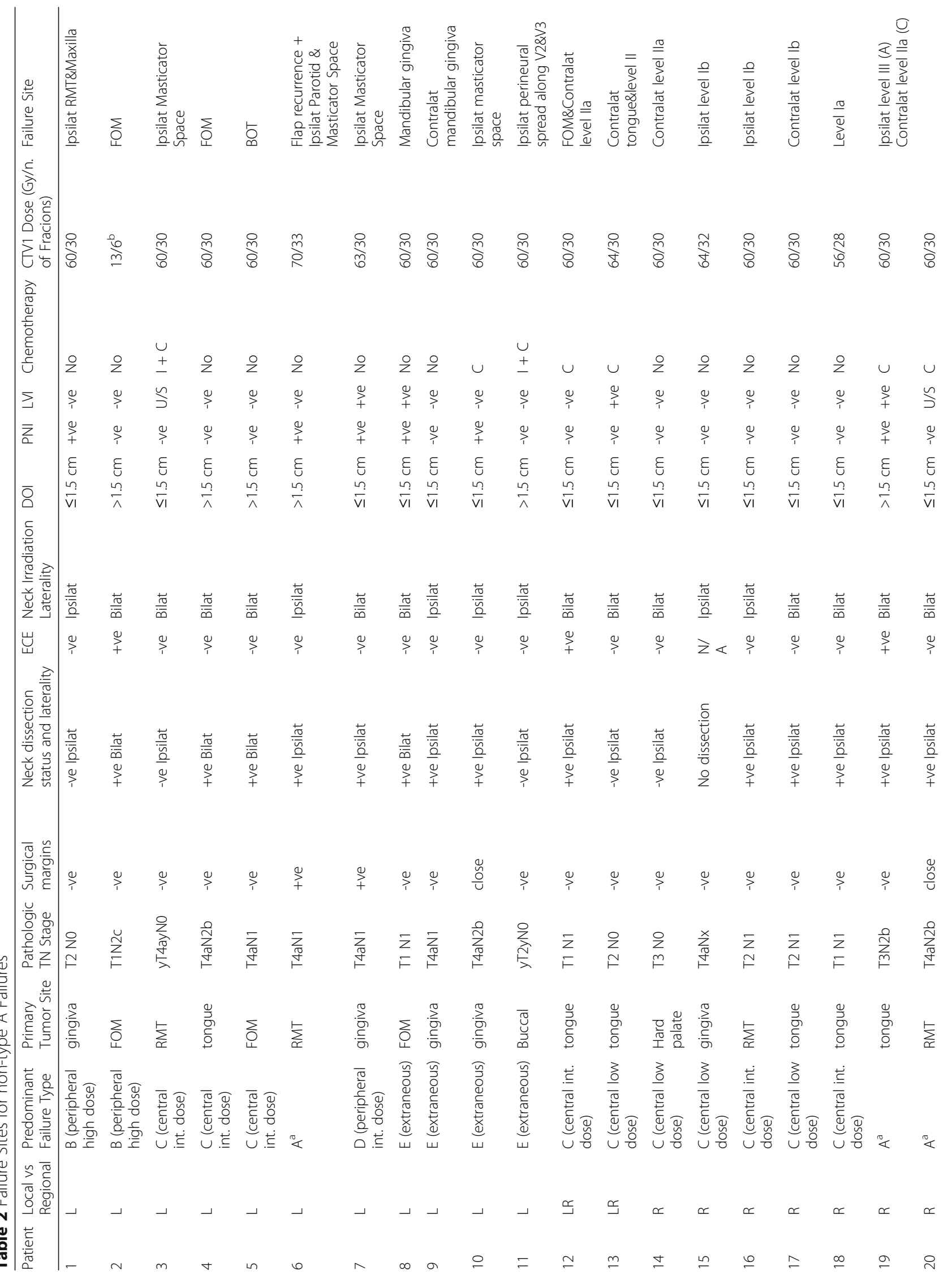




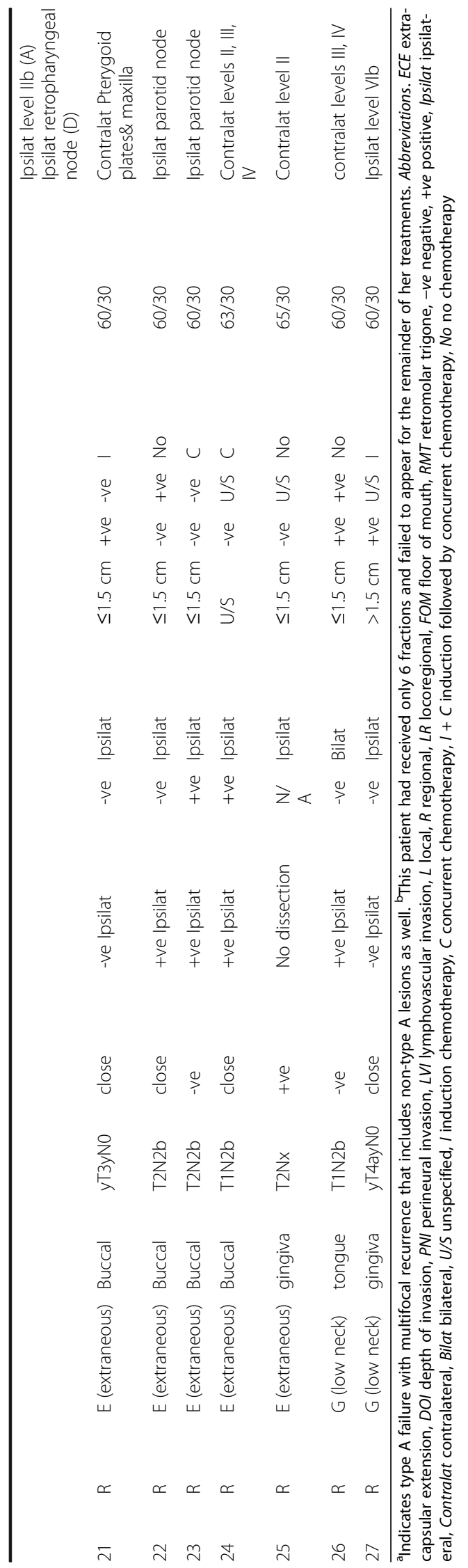



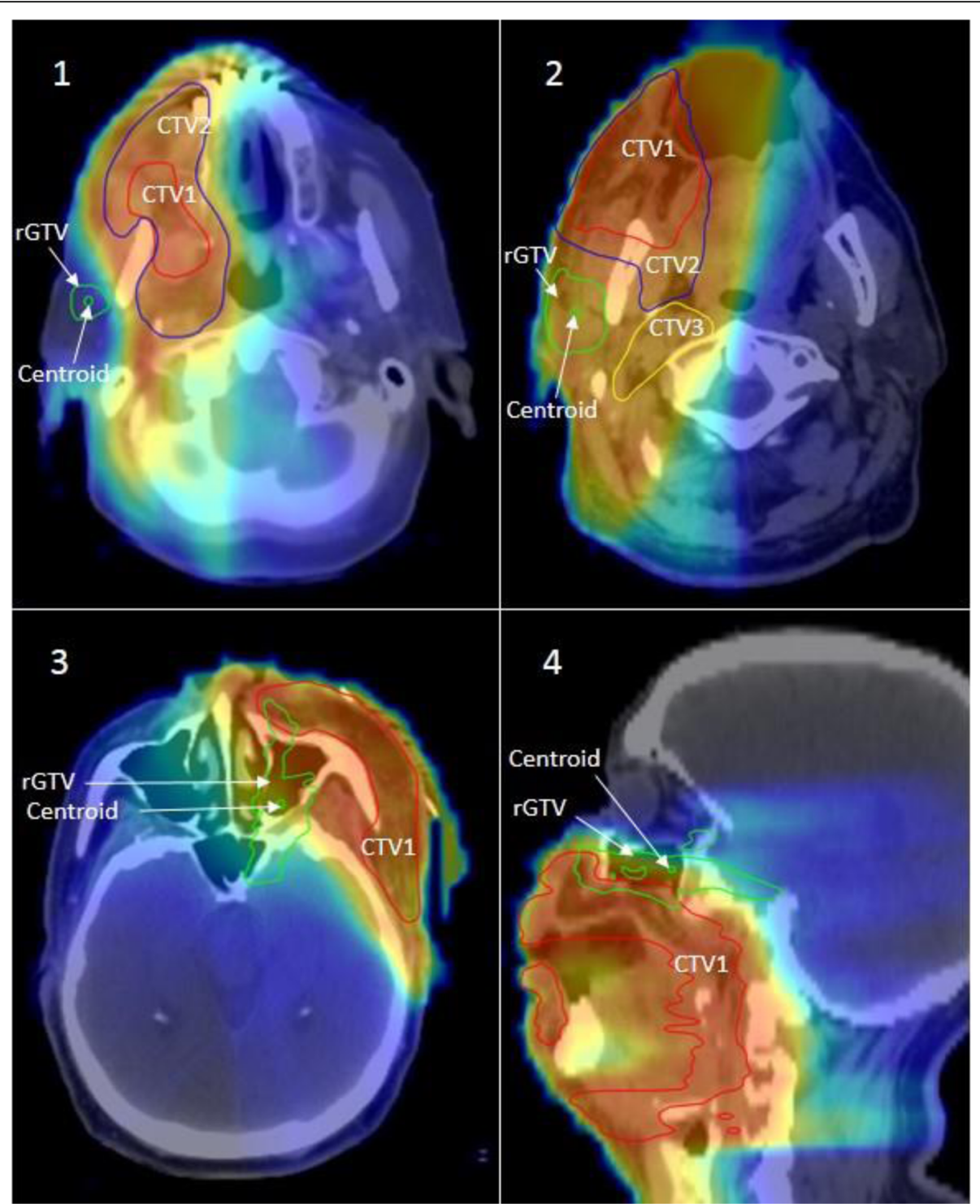

Fig. 4 The top panel depicts two patients with type E recurrence in the ipsilateral parotid nodal area following parotid sparing IMRT. Both patients were diagnosed with T2N2b right sided buccal mucosa primaries and subsequently failed at the ipsilateral parotid area outside all target volumes. The lower panel depicts another example of type E failure in a patient diagnosed with T3N2b at the buccal mucosa with post-IMRT ipsilateral perineural spread along the maxillary and mandibular nerves (bottom left, bottom right)

majority of which were in the high dose tumor bed target (Type A), while the remainder were in subclinical (Type $\mathrm{C}$ and $\mathrm{G}$ ) targets. No technical or operatordependent processes could conceivably prevent such failures. Whether dose intensification to Type A targets would minimize these recurrences is unclear. To date, the benefits of treatment intensification seem small. There is a paucity of data demonstrating that increasing radiation dose is beneficial, and even concurrent chemoradiation only seems to benefit those at highest risk $[5,18]$. Type $C$ failures may be prevented by prescribing higher doses (i.e. shifting to higher TV levels), but it is unclear if these relatively small dose increments (as the differences between dose to each CTV was $<10 \%$ ) would be beneficial, and also increasing dose to larger volumes can potentially increase the risk: benefit ratio. Non-IMRT failure in the matching low-neck supraclavicular field was very uncommon and only seen in two patients. Also, no failures were noted at the IMRT-supraclavicular field match-line confirming the safety of this technique.

Types B (peripheral high dose), D (peripheral elective dose) and E (extraneous) failure are potentially dependent on technical or radiotherapy processes. Type $B$ and $D$ recurrences are analogous to what has been described as "marginal miss". Peripheral misses (type B) were seen only in 4 patients (of whom one didn't 
complete the prescribed radiation dose). Three of these were in primary tumor sites, and more likely reflect the tumors finding pathways more amenable for spread, and growing out of the dose region rather than originating at the periphery. Similarly, the one peripheral nodal failure was in a retropharyngeal node that is typically not targeted, but fell into a D type failure rather than $\mathrm{E}$ due to the proximity to the primary tumor bed. The paucity of these peripheral type recurrences reflects an adequate CTV delineation strategy, appropriate PTV margins, and precise dose delivery.

Type E extraneous recurrences were seen in approximately $17 \%$ of failures. Type E failures are analogous to traditionally defined "out of field" recurrences. This pattern of failure was mainly in patients who had primary tumors of the buccal mucosa or gingiva. Four patients had primary type E recurrence, and 5 had nodal type E recurrences. Two patients with type $E$ failure at the primary site had recurrences in sites relatively separate from the primary disease, and so these "recurrences" may represent second primary tumors.

Retrograde ipsilateral perineural spread was the cause of Type E recurrence in two patients (Fig. 4), and was also observed in two other patients ( 1 Type $C$ and 1 Type D) as seen in Table 2. Daly et al. had reported on a patient who had developed failure at the ipsilateral masseter due to presence of perineural invasion and retrograde tracking along the mandibular nerve [11]. Yao et al. had previously reported on two patients with extensive perineural invasion and retrograde tracking who had developed failure within the infratemporal fossa [8]. We would also recommend that "nerves at risk in the tumor bed should undergo biopsy and be covered in a retrograde fashion within the RT field" [11].

Three patients had recurrence in the contralateral undissected/unirradiated upper neck. Prior studies have reported that positive ipsilateral lymph nodes are a predictive factor for contralateral recurrence; conversely, contralateral lymph node metastases never occurred in patients without ipsilateral lymph node involvement $[19,20]$. While these studies demonstrated the association of ipsilateral lymph node involvement with contralateral recurrence, the majority of patients in these studies were predominantly patients with oral tongue cancer, and few patients had buccal cancer. We continue to favor comprehensive bilateral radiation for patients with tumors in central oral cavity sites, such as the oral tongue and floor of mouth. Yao et al. recommended that patients with ipsilateral lymph node involvement in OCC should receive bilateral neck irradiation [8]. Moreover, Chan et al. suggested that bilateral neck irradiation should be administered to patients with N2b disease [13]. However, again these studies were heavily weighted with patients with oral tongue, and not buccal cancers. Thus our approach to buccal and retromolar trigone tumors is individualized. Those patients with low nodal burden are still treated to the ipsilateral neck, but those with bulky nodes, multiple levels of nodes, or who have an epicentered lateral primary site but the primary disease extends centrally are treated to both sides of the neck.

Two patients had almost identical pattern of recurrence in the ipsilateral parotid nodes as shown in Fig. 4. Strict dose constraints to the parotid have been recommended to avoid long-term risks of xerostomia [10]. In our cohort, two patients with T2N2b buccal mucosa primaries had recurrences in the ipsilateral parotid gland which was spared during PO-IMRT. This phenomena has been also reported in previous studies [13]. The proximity of buccal and retromolar trigone tumors to the parotid bed makes ipsilateral parotid avoidance challenging. We therefore recommend limiting the extent of radiation-induced xerostomia by focusing on sparing the contralateral glands.

To date, a limited number of studies have exclusively investigated failure following PO-IMRT in OCC patients. Additional file 1: Tables S1 and S2 tabulate our study's patient/treatment and failure characteristics compared to extant literature. Loco-regional control of our study are consistent with that of previous studies [8-13, 21]. Although Sher et al. reported low loco-regional failure rates $(7 \%)$, they acknowledged that it may have reflected the greater proportion of early $\mathrm{T}$ and $\mathrm{N}$ staging in their cohort [12]. Other disease characteristics are noted but are not directly comparable as the subset of reported patients varied from study to study.

To classify failures using a spatial component, several prior studies $[8,9,11-13]$ used varying volume overlap approaches [22-24]. Here we highlight two limitations in these prior studies: 1) volume overlap methods for spatial characterization and 2) the lack of a dosimetric component in failure analysis. Given enough time, recurrence volumes can outgrow TV margins. Thus, the spatial characterization of "infield" vs. "outfield" is volume dependent and biased by elapsed time. As the spatial component of our failure classification, we apply a centroid-based approach. This approach has demonstrated to be more superior and accurate than volumetric overlap approaches, as the latter tends to assign failures more peripherally $[25,26]$. Moreover, the spatial component alone is insufficient for accurate and specific reporting of failures. Without a dosimetric component, failures that are "infield" but in fact did not receive the prescribed dose (i.e. Types B and D in our classification) could be erroneously assumed to be biological failures. Subsequently, such "infield" failures are not investigated further despite a potentially rectifiable technical or radiotherapy process. 
As a retrospective series, the standard caveats apply. However, although patients were not selected nor treated prospectively, all patients were reviewed by a multidisciplinary team. This data was collated as a secondary analysis of part of a larger programmatic evaluation of PO-IMRT outcomes for OCC; the reader is encouraged to peruse the clinical/oncologic report previously published [17]. Likewise, as a single institute series from a high-volume tertiary center, the generalizability/scalability of our findings to facilities which do not utilize our systematic quality assurance methods (e.g. multi-physician direct physical examination and consensus review of target delineation) would be suspect $[27,28]$.

Nevertheless, despite these limitations, our study is the largest, to our knowledge, systematic assessment of patterns of failure to OCC following PO-IMRT using a qualitycontrolled image-registration pipeline for methodologically rigorous pattern of failure investigation [16]. Similarly, our study is the first to incorporate a dosimetric component in failure classification for OCC following PO-IMRT, in addition to utilizing a centroid-based spatial component and a validated DIR method which is critical for accurate failure analysis [14-16]. We hope that by utilizing a standardized typology for reporting patterns of failure in OCC following PO-IMRT, which can be adopted by multiple institutions, we can encourage other comparable reporting practices for PO-IMRT, in a manner that allows improved detection of possible modes of preventable error. This could allow for pooling of data to infer differences in treatment approaches and subsequent outcomes amongst different institutions.

\section{Conclusions}

Prior studies have assessed loco-regional control following PO-IMRT to OCC in manner which elides the reality of dosimetric gradients inherent in IMRT, and precludes identification of systematic sources of modifiable error which might impact these recurrences. A standardized typology with both spatial and dosimetric components allows for more accurate and specific reporting of the patterns of failure over traditional "infield" vs. "marginal" vs. "outfield" failure classification schemes. Our study incorporates a dosimetric component in addition to utilizing a centroidbased spatial component and a quantitatively validated DIR method. Approximately half of the patients with local and/or regional failure included in the current study had non-central high dose recurrence. Thus, contrary to nonOCC sites, a substantial proportion of failures in our series, despite rigorous multiphysician quality assurance, are not definitive biological failures and, as potentially modifiable risk-events, necessitate further investigation and potential practice modification. Other groups are encouraged to undertake similar efforts as single-site or pooled analyses for OCC following PO-IMRT.

\section{Additional file}

Additional file 1:Tables S1 and S2. Showing our study's patient/ treatment and failure characteristics compared to extant literature. (PDF 99 kb)

\section{Abbreviations}

DIR: Deformable image registration; fD95\%: dose to 95\% of the failure volume; IRB: Institutional review board; $\mathrm{PCT}$ : planning $C T$; PO-IMRT: Postoperative intensity modulated radiotherapy; rCT: recurrence CT; rGTV: recurrence gross tumor volume; ROls: Regions of interest; TVs: Target volumes

\section{Acknowledgments}

Not applicable.

\section{Funding}

Drs. Lai, Mohamed, and Fuller receive funding support from the National Institutes of Health (NIH)/National Institute for Dental and Craniofacial Research (1R01DE025248-01/R56DE025248-01). Dr. Fuller is a Sabin Family Foundation Fellow and receives grant and/or salary support from the NIH/National Cancer Institute (NCl) Head and Neck Specialized Programs of Research Excellence (SPORE) Developmental Research Program Award (P50CA097007-10) and Paul Calabresi Clinical Oncology Program Award (K12 CA088084-06); the National Science Foundation (NSF), Division of Mathematical Sciences, Joint NIH/NSF Initiative on Quantitative Approaches to Biomedical Big Data (QuBBD) Grant (NSF 1557679); the NIH Big Data to Knowledge (BD2K) Program of the National Cancer Institute (NCl) Early Stage Development of Technologies in Biomedical Computing, Informatics, and Big Data Science Award (1R01CA214825-01); NCI Early Phase Clinical Trials in Imaging and Image-Guided Interventions Program (1R01CA21814801); a General Electric Healthcare/MD Anderson Center for Advanced Biomedical Imaging In-Kind Award; an Elekta AB/MD Anderson Department of Radiation Oncology Seed Grant; the Center for Radiation Oncology Research (CROR) at MD Anderson Cancer Center Seed Grant; and the MD Anderson Institutional Research Grant (IRG) Program. Dr. Fuller has received speaker travel funding from Elekta AB. Supported in part by the National Institutes of Health $(\mathrm{NIH}) /$ National Cancer Institute (NCI) Cancer Center Support (Core) Grant CA016672 to The University of Texas MD Anderson Cancer Center.

\section{Availability of data and materials}

Please contact the corresponding authors for data requests.

\section{Authors' contributions}

All listed co-authors performed the following: 1. Substantial contributions to the conception or design of the work; or the acquisition, analysis, or interpretation of data for the work; 2. Drafting the work or revising it critically for important intellectual content; 3. Final approval of the version to be published; 4. Agreement to be accountable for all aspects of the work in ensuring that questions related to the accuracy or integrity of any part of the work are appropriately investigated and resolved. Specific additional individual cooperative effort contributions to study/manuscript design/execution/interpretation, in addition to all criteria above are listed as follows: ASRM and AJW- Undertook clinical and imaging data collection; direct oversight of all image registration/segmentation, and data collection workflow, drafted initial manuscript, and participated in data analysis and interpretation of data. CDF- Co-primary investigator; with ASG conceived project and interpreted study results, direct and final oversight of imaging and clinical data collection. AJW, MK, GBG, JP, WHM, BMB, HS, SYL, SRQ, AMB, SJF, DIR, AGE - Direct patient care provision, direct imaging assessment and clinical data collection; interpretation and analytic support. ASG-Corresponding author; co-primary investigator; conceived, coordinated, and directed all study activities, responsible for data collection, project integrity, manuscript content and editorial oversight and correspondence. All authors read and approved the final manuscript.

\section{Ethics approval and consent to participate}

This study was initiated after the approval of the institutional review board at The University of Texas, MD Anderson Cancer Center of the study protocol \#PA12-0168.

Consent for publication

Not applicable.

Competing interests

The authors declare that they have no competing interest. 


\section{Publisher's Note}

Springer Nature remains neutral with regard to jurisdictional claims in published maps and institutional affiliations.

\section{Author details}

'Departments of Radiation Oncology, The University of Texas MD Anderson Cancer Center, Unit 97, 1515 Holcombe Boulevard, Houston, TX 77030, USA. ${ }^{2}$ Department of Head and Neck Surgery, The University of Texas MD Anderson Cancer Center, Unit 97, 1515 Holcombe Boulevard, Houston, TX 77030, USA. ${ }^{3}$ Department of Clinical Oncology and Nuclear Medicine, Faculty of Medicine, University of Alexandria, Alexandria, Egypt. ${ }^{4}$ Department of Clinical Oncology and Nuclear Medicine, Faculty of Medicine, University of Ain-Shams, Cairo, Egypt. ${ }^{5}$ Department of Radiation Oncology, Allentown Radiation Oncology Associates, Allentown, PA, USA.

Received: 7 July 2017 Accepted: 9 August 2017

Published online: 15 August 2017

\section{References}

1. Huang DT, Johnson CR, Schmidt-Ullrich R, Grimes M. Postoperative radiotherapy in head and neck carcinoma with extracapsular lymph node extension and/or positive resection margins: a comparative study. Int J Radiat Oncol Biol Phys. 1992;23:737-42.

2. Ang KK, Trotti A, Brown BW, Garden AS, Foote RL, Morrison WH, et al. Randomized trial addressing risk features and time factors of surgery plus radiotherapy in advanced head-and-neck cancer. Int J Radiat Oncol Biol Phys. 2001;51:571-8

3. Robertson AG, Soutar DS, Paul J, Webster M, Leonard AG, Moore KP, et al. Early closure of a randomized trial: surgery and postoperative radiotherapy versus radiotherapy in the management of intra-oral tumours. Clin Oncol. 1998;10:155-60

4. Boero IJ, Paravati AJ, Xu B, Cohen EEW, Mell LK, Le Q-T, et al. Importance of radiation oncologist experience among patients with head-and-neck cancer treated with intensity-modulated radiation therapy. J Clin Oncol. 2016;34:684-90.

5. Blanchard P, Baujat B, Holostenco V, Bourredjem A, Baey C, Bourhis J, et al. Meta-analysis of chemotherapy in head and neck cancer (MACH-NC): a comprehensive analysis by tumour site. Radiother Oncol. 2011;100:33-40.

6. Daly ME, Le QT, Kozak MM, Maxim PG, Murphy JD, Hsu A, et al. Intensitymodulated radiotherapy for oral cavity Squamous cell carcinoma: patterns of failure and predictors of local control. Int J Radiat Oncol. 2011;80:1412-22.

7. Ooishi M, Motegi A, Kawashima M, Arahira S, Zenda S, Nakamura N, et al. Patterns of failure after postoperative intensity-modulated radiotherapy for locally advanced and recurrent head and neck cancer. Jpn J Clin Oncol. 2016;46:919-27.

8. Yao M, Chang K, Funk GF, Lu H, Tan H, Wacha J, et al. The failure patterns of oral cavity squamous cell carcinoma after intensity-modulated radiotherapy-the university of iowa experience. Int J Radiat Oncol Biol Phys. 2007;67:1332-41.

9. Studer G, Zwahlen RA, Graetz KW, Davis BJ, Glanzmann C. IMRT in oral cavity cancer. Radiation Oncology (London, England). 2007; 2:16.

10. Gomez DR, Zhung JE, Gomez J, Chan K, Wu AJ, Wolden SL, et al. Intensitymodulated radiotherapy in postoperative treatment of oral cavity cancers. Int J Radiat Oncol Biol Phys. 2009;73:1096-103.

11. Daly ME, Le Q-T, Kozak MM, Maxim PG, Murphy JD, Hsu A, et al. Intensitymodulated radiotherapy for oral cavity Squamous cell carcinoma: patterns of failure and predictors of local control. Int J Radiat Oncol Biol Phys. 2011;80:1412-22.

12. Sher DJ, Thotakura V, Balboni TA, Norris CM Jr, Haddad RI, Posner MR, et al. Treatment of oral cavity Squamous cell carcinoma with adjuvant or definitive intensity-modulated radiation therapy. Int J Radiat Oncol Biol Phys. 2011;81:e215-e22.

13. Chan AK, Huang SH, Le LW, Yu E, Dawson LA, Kim JJ, et al. Postoperative intensity-modulated radiotherapy following surgery for oral cavity squamous cell carcinoma: patterns of failure. Oral Oncol. 2013;49:255-60.

14. Gunn GB, Blanchard P, Garden AS, Zhu XR, Fuller CD, Mohamed AS, et al. Clinical outcomes and patterns of disease recurrence after intensity modulated proton therapy for Oropharyngeal Squamous carcinoma. Int J Radiat Oncol Biol Phys. 95:360-7.

15. Mohamed AS, Awan M, Kocak E, Beadle BM, Kantor ME, Gunn GB, et al. Methods for analysis and reporting the patterns of Locoregional failure in the era of IMRT for head and neck cancer: deformable image registration-based quality assurance workflow. Int J Radiat Oncol Biol Phys. 2014;90:S569-S70.
16. Mohamed ASR, Rosenthal DI, Awan MJ, Garden AS, Kocak-Uzel E, Belal AM et al. Methodology for analysis and reporting patterns of failure in the era of IMRT: head and neck cancer applications. Radiat Oncol. 2016;11:1-10.

17. Quinlan-Davidson SR, Mohamed ASR, Myers JN, Gunn GB, Johnson FM, Skinner $\mathrm{H}$, et al. Outcomes of oral cavity cancer patients treated with surgery followed by postoperative intensity modulated radiation therapy. Oral Oncol. 2017;72:90-7.

18. Pignon JP, le Maitre A, Maillard E, Bourhis J, Group M-NC. Meta-analysis of chemotherapy in head and neck cancer (MACH-NC): an update on 93 randomised trials and 17,346 patients. Radiother Oncol: J Eur Soc Therapeut Radiol Oncol. 2009:92:4-14.

19. Chow TL, Chow TK, Chan TTF, Yu NF, Fung SC, Lam SH. Contralateral neck recurrence of squamous cell carcinoma of oral cavity and oropharynx. J Oral Maxillofac Surg. 2004;62:1225-8.

20. Kurita H, Koike T, Narikawa J-N, Sakai H, Nakatsuka A, Uehara S, et al. Clinical predictors for contralateral neck lymph node metastasis from unilateral squamous cell carcinoma in the oral cavity. Oral Oncol. 2004;40:898-903.

21. Metcalfe E, Aspin L, Speight R, Ermis E, Ramasamy S, Cardale K, et al. Postoperative (chemo) radiotherapy for oral cavity Squamous cell carcinomas: outcomes and patterns of failure. Clin Oncol (R Coll Radiol). 2017:29:51-9.

22. Chao KSC, Ozyigit G, Tran BN, Cengiz M, Dempsey JF, Low DA. Patterns of failure in patients receiving definitive and postoperative IMRT for head-andneck cancer. Int J Radiat Oncol Biol Phys. 2003;55:312-21.

23. Popovtzer A, Gluck I, Chepeha DB, Teknos TN, Moyer JS, Prince ME, et al. The pattern of failure after Reirradiation of recurrent Squamous cell head and neck cancer: implications for defining the targets. Int J Radiat Oncol Biol Phys. 2009;74:1342-7.

24. Dawson LA, Anzai Y, Marsh L, Martel MK, Paulino A, Ship JA, et al. Patterns of local-regional recurrence following parotid-sparing conformal and segmental intensity-modulated radiotherapy for head and neck cancer. Int J Radiat Oncol Biol Phys. 2000;46:1117-26.

25. Due AK, Vogelius IR, Aznar MC, Bentzen SM, Berthelsen AK, Korreman SS, et al. Methods for estimating the site of origin of locoregional recurrence in head and neck squamous cell carcinoma. Strahlenther Onkol. 2012;188:671-6.

26. Raktoe SAS, Dehnad H, Raaijmakers CPJ, Braunius W, Terhaard CHJ. Origin of tumor recurrence after intensity modulated radiation therapy for Oropharyngeal Squamous cell carcinoma. Int J Radiat Oncol Biol Phys. 2013;85:136-41.

27. Rosenthal DI, Asper JA, Barker JL, Garden AS, Chao KSC, Morrison WH, et al. Importance of patient examination to clinical quality assurance in head and neck radiation oncology. Head Neck. 2006;28:967-73.

28. Cardenas CE, Mohamed ASR, Tao R, Wong AJR, Awan MJ, Kuruvila S, et al. Prospective qualitative and quantitative analysis of real-time peer review quality assurance rounds incorporating direct physical examination for head and neck cancer radiation therapy. Int J Radiat Oncol Biol Phys. 2017:98: $532-40$

\section{Submit your next manuscript to BioMed Central and we will help you at every step:}

- We accept pre-submission inquiries

- Our selector tool helps you to find the most relevant journal

- We provide round the clock customer support

- Convenient online submission

- Thorough peer review

- Inclusion in PubMed and all major indexing services

- Maximum visibility for your research

Submit your manuscript at www.biomedcentral.com/submit
Biomed Central 